See discussions, stats, and author profiles for this publication at: https://www.researchgate.net/publication/305791193

\title{
Online inspection system based on machine learning techniques: real case study of fabric textures classification for the automotive industry
}

Article in Journal of Intelligent Manufacturing • August 2016

DOI: 10.1007/s10845-016-1254-6

\section{CITATIONS}

9

5 authors, including:

Pedro Malaca

University of Porto

9 PUBLICATIONS 32 CITATIONS

SEE PROFILE

Joao Silva

Cooperativa de Ensino Superior, Politécnico e Universitário

6 PUBLICATIONS 17 CITATIONS

SEE PROFILE
189

Luís F. Rocha

Institute for Systems and Computer Engineering, Technology and Science (INESC ... 34 PUBLICATIONS 186 CITATIONS

SEE PROFILE

(3) Germano Veiga

Institute for Systems and Computer Engineering, Technology and Science (INESC ... 83 PUBLICATIONS 356 CITATIONS

SEE PROFILE

Some of the authors of this publication are also working on these related projects:

Robotic Bin Picking of Entangled Tubes View project

Robotic Technologies for Non-Standard Design and Construction in Architecture (RobTech) View project 


\title{
Online Inspection System based on Machine Learning Techniques: Real case study of fabric textures classification for the automotive industry
}

\author{
Pedro Malaca • Luis F. Rocha • D. \\ Gomes · João Silva • Germano Veiga
}

Received: date / Accepted: date

\begin{abstract}
This paper focus on the classification, in real-time and under uncontrolled illumination, of fabric textures for the automotive industry. Many industrial processes do not permit effective control of illumination of their vision based systems, hindering their effectiveness. The ability to overcome these problems using classification methods with suitable pre-processing techniques and choice of characteristics will allow to increase the efficiency of this type of solutions with obvious production gains and thus economical. For this purpose, in this paper was studied and analyzed various pre-processing techniques, and were also selected the most appropriate fabric characteristics for the considered industrial case scenario. The methodology followed was based on the comparison of two different machine learning classifiers, ANN and SVM, using a large set of samples with great variability of illumination conditions in order to faithfully simulate the industrial environment. The obtained solution shows the sensibility of ANN over SVM in respect to the number of features and the size of the training set, showing the better effectiveness and robustness of the last. The characteristics vector uses histogram equalization, Laws filter and Sobel filter, and multi-scale analysis. By using a correlation based

\author{
Pedro Malaca \\ Electrical and Computer Engineering Department, University of Porto, Portugal \\ SARKKIS Robotics, Lda., Porto, Portugal \\ E-mail: pedro.malaca@sarkkis.com \\ Luis F. Rocha \\ INESC-TEC, INESC Technology and Science, Porto, Portugal \\ D. Gomes \\ Mechanical Engineering Department, University of Coimbra, Portugal \\ João Silva \\ SARKKIS Robotics, Lda., Porto, Portugal \\ Germano Veiga \\ INESC-TEC, INESC Technology and Science, Porto, Portugal
}


method was possible to reduce the number of features used, achieving a better balanced between processing time and classification ratio.

Keywords Computer vision · Perception and Recognition · Fabric Analyses · Machine Learning · Uncontrolled Illumination · Automotive Industry

\section{Introduction}

Today's industrial manufacturing environment, and driven by the current market globalization, has taking into consideration competitive factors, such as time, cost and quality of the products fabricated. Furthermore, products are more and more characterized by customization and increased complexity. The conjugation of both of these factors, leads to the need of companies to be able to continuously and quickly adapt all entities inherent to manufacturing systems, namely the ones related to manufacturing resources, materials, and information flows [35].

Nowadays, and due to the constant development of technology, sophisticated machinery is increasingly available, helping companies to achieve a significant increase in production process control, guarantying products quality and reducing machinery set-up time.

In this context, the growing of real-time artificial vision systems with low computation cost has been a good promoter for its introduction in industrial production systems. This type of technology is in our days being explored in diversified fields of application like sensing, inspection [1] or even perception $[2,3]$ and learning [5-7], resulting on its increasing importance in automation systems of the future and providing tools for better production technologies and quality control.

In the textile industry the recognition of fabric textures is usually made manually. The little automation effectively used normally achieves poor results mostly due to uncontrolled light conditions that severely contribute for reducing the systems efficiency.

Considering this machine vision drawback, this paper addresses an automotive industrial case scenario where the current system used for fabric textures recognition (Figure 1) presents poor results, showing that is not selfsustainable. Hence, it becomes necessary to provide the system with extra tools to improve its reliability and robustness. To achieve this goal different machine learning methods with different combinations of features were explored and applied in the industrial cell. With the development of this system becomes possible the early detection of fabric textures nonconformities on the assembled products, with high degree of reliability and robustness.

Today, lighting is still one of the most critical factors that influence the success of a machine vision system, having direct impact on the accuracy, reliability and response time of the same $[8,9]$. As a result many machine vision applications adopt/require solutions to condition the ambient light $[3$, 9]. Therefore, the ability to overcome the uncontrolled illumination issue with 


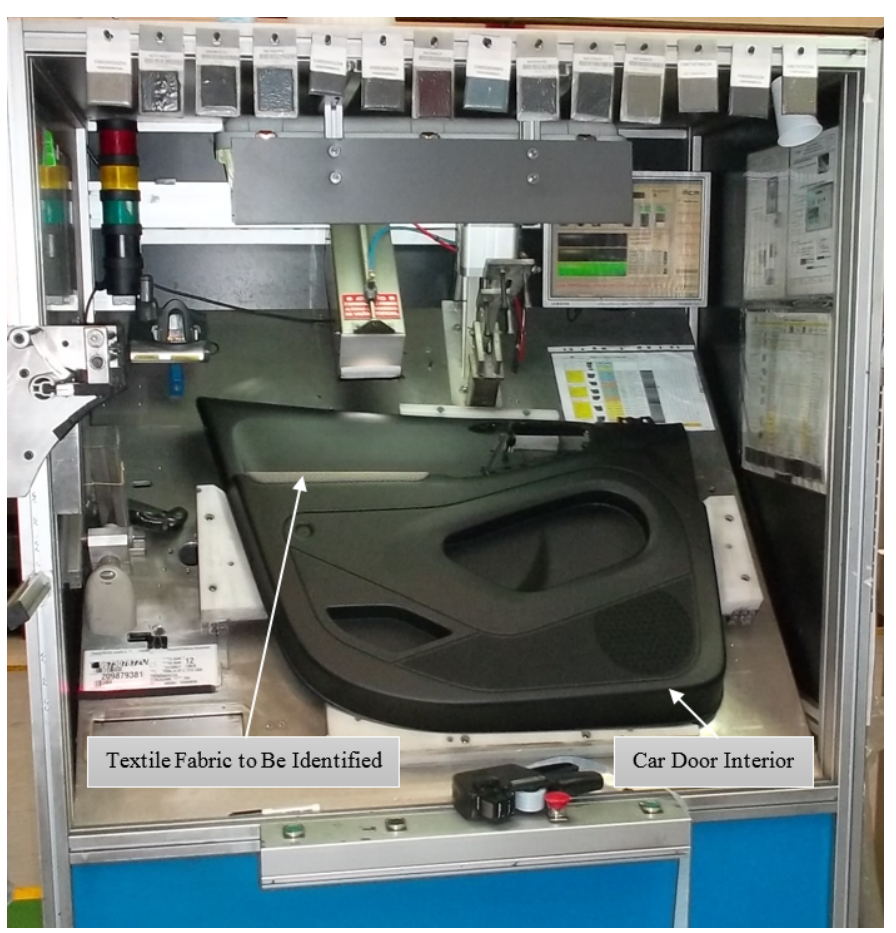

Fig. 1 The considered industrial work cell. It consists in an artificial based vision system used for quality control of fabrics on doors interiors in the automotive industry.

suitable techniques of pre-processing and choice of specific image characteristics will allow to increase the efficiency and reliability of machine vision based identification systems, with obvious production gains and thus economical.

\section{PROBLEM STATEMENT}

\subsection{Challenge}

The automotive industry is very peculiar, due to the products and the market nature. A car is a rather complex product to build, with short life-cycles, mass produced, and with very high quality requirements. Therefore, the suppliers of the automotive industry combine short product life-cycles, with high throughput seasonal demands and very restrict quality control. For example, the automotive plastics industry produce the parts just-in-time and directly customized to the car being produced in the final automotive assembly line, that can be located hundreds or thousands of kilometers away.

Simoldes Plásticos ${ }^{1}$ currently assembles car doors interiors, in which uses a component produced with textile-in-mold injection. This small component

\footnotetext{
1 http://www.simoldes.com/plastics/
} 
is assembled in the door interior part, also in plastic, and before the shipment to the client the color/pattern of the fabric must be checked against the reference in the bigger part. Due to the nature of the quality control machine is not possible to have a complete dark set-up vision system, which makes the system used nowadays (based on color analysis) very sensitive to the ambient illumination.

\subsection{Objectives}

Considering the problem described, the objective of this work is to create a robust artificial vision based system for automatic fabric texture identification that meet the industrial requirements: the algorithm must be computationally fast; and the recognition rate must be close to $100 \%$.

Having this objective in mind, this work is divided in two parts: firstly are explored two different machine learning approaches while applying different image processing methods to extract the necessary features; and secondly the use of algorithms that perform the best selection of the features and analyze the computation cost and efficiency of each learning method in order to achieve the best solution.

\subsection{Laboratory Work Cell and Data Set}

To test the robustness of the algorithms is necessary a high number of images in different light conditions and in different positions considering all possible types of fabric textures.

In this particular test case were considered eight different classification classes, visible in the Figure 2. During one month were collected images of this texture fabrics in the Robotics Laboratory of the Mechanical Department in University of Coimbra. This laboratory has an open space with multiple windows and no shuttles recreating the factory conditions, i.e., an industrial shoop floor with no control of the environment light.

To collect the required images and simulate the desired functionality a work cell was created (Figure 3), consisting on a software application that interacts with an industrial robot and a CCD camera placed on the robots end effector (eye-in-hand). The application gives order to the robot to move to the first position. When it reaches the required position, it gives a signal to the application to collect the image of the first fabric texture (to acquire the images was used an application based on Aforge.NET [23]). After performing the first collection the application gives order to the robot to move to the second position. This task is repeated for all eight fabric textures. In each cycle the application collects eight images. Moreover, to increase the robustness of the application, namely invariant to parts that were placed upside down, in each cycle the robot rotates the camera one hundred and eighty degrees.

Figure 4 presents an example of one of the considered fabric textures (class 1) under different weather and environment lighting conditions. The natural 




Fig. 2 Fabric textures classes from Simoldes Plásticos production line.

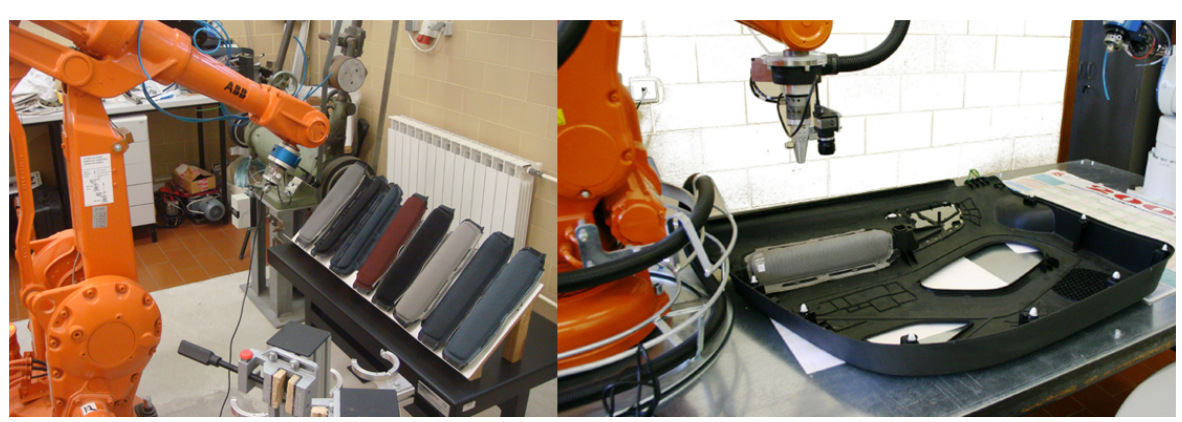

Fig. 3 Laboratory work cell set-up.

weather information was extracted from a weather forecast internet site in the beginning of each trajectory cycle of the robot.

Furthermore, a Human-Machine Interface was also developed. The created interface can be divided into two parts: the first to perform the classification and show the results to the operator; a second to give the possibility to improve the classifier by training the same. The second tab of the application was used to show the previous saved images, taken during the execution of the system, and where the operator choose the correct class label of the sample. After this, the classifier is retrained and will be more precise the next day of work. 


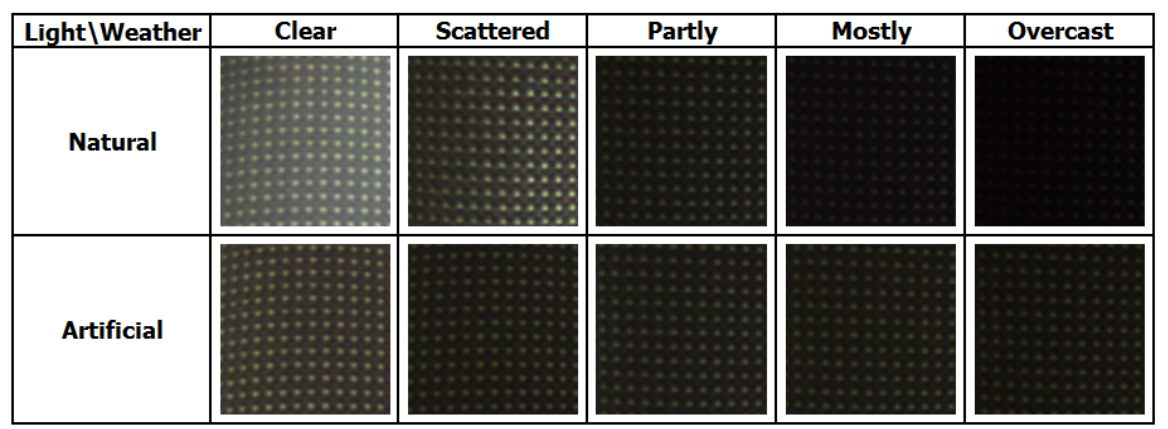

Fig. 4 Images example of one of the fabric textures (Class 1) under different weather and uncontrolled environment light conditions.

\section{MACHINE LEARNING METHODS}

In the literature is possible to find different methods to solve a classification problem [11-15]. Each method claim its advantage depending on the application in study, and is inconclusive that one method is better than all the others. Therefore, to create a robust algorithm 1880 images were collected and analyzed using 1424 for classifiers training purpose and the other 456 for testing. The distribution of this data set considering the outdoor weather ("clear","scattered","partly","mostly","overcast") and the room light condition ("natural" or with "artificial" light) is presented in Figure 5.

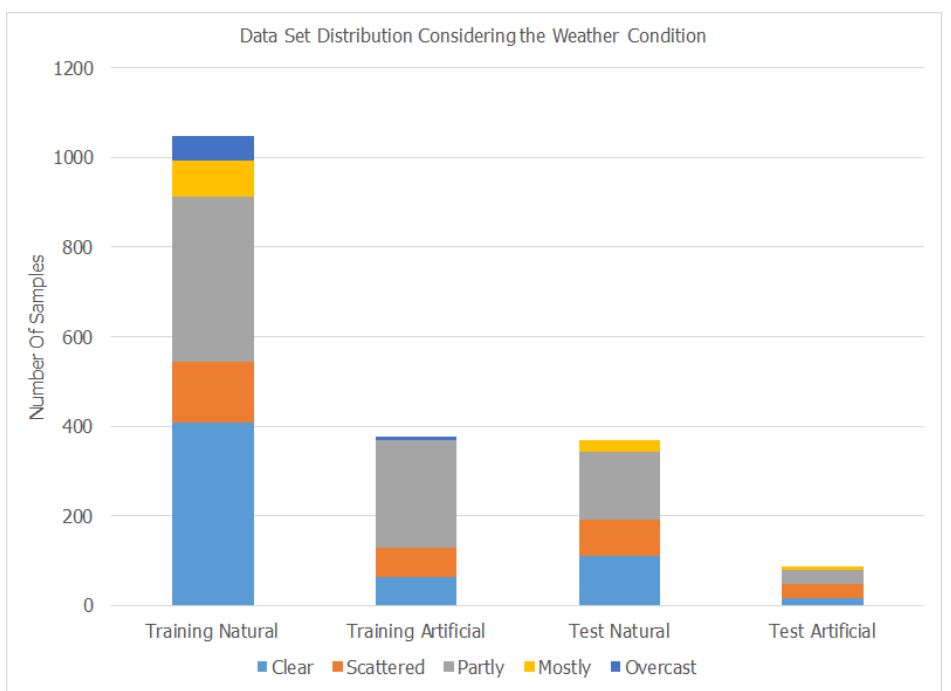

Fig. 5 Graph showing the distribution of the train and test images by the weather condition that was verified when the image was extracted. 
The classifiers explored were Support Vector Machine (SVM) [37] and Artificial Neural Networks (ANN) [36], using the implementation available in MATLAB software. These two methods have been widely used and explored in different areas of application [16-20]. Furthermore, they have been applied successfully in past textile industry applications $[13,14]$.

A huge amount of tests were performed to reach the best classifier model, especially in what refers to the ANN classifier due to its dependency on the initial weights. Based on the best practice shown on [21], these weights were randomly initialized in each test, and in the end were obtained the most reliable values for the presented case study.

In what refers to the ANN model, the configuration selected was a single network with 4 neurons in the hidden layer, with a sigmoid transfer function, and a linear transfer function in the output layer. Moreover, for training the neural network was used the Levenberg method with a learning rate of 0.1 and the mean square error as performance evaluation. In the case of SVM the best model was obtained using the Sequential minimal optimization (SMO) method with a third order polynomial kernel and a cost equal to 1.5.

In the solution adopted was decided to use supervised instead of unsupervised learning, since the first is indicated for cases where the categories/classes of the data are known in advance. This is the case of the industrial case study presented in this paper where all types of fabrics to be classified are clear defined in advance.

For its turn, unsupervised learning refers to techniques that find patterns in unlabeled data. In this case, in the beginning there are no clear defined classes, and the learning process attempts to find appropriate categories/clusters for the input data.

\section{FEATURES EXTRACTION}

Focusing on achieving the best classification results was explored a diversity of image processing techniques to, by one hand reduce the light changing effects and, on the other highlight the characteristics of the fabric textures. Due to the need of parameterization and optimization in the pre-processing stage, which allows a high number of combinations, this study turns to be very extensive. Therefore, to simplify the perception of the developed work this section starts by performing an introduction of the selected features by steps, showing also the classification result achieved in each phase.

\subsection{Models of Colors and Histogram}

The principal methods for extracting fabric texture features can be classified as statistical approaches, structural, and spectral [24,25]. This work focuses on the first, since the second is considered appropriate when the primitive fabric texture is large enough to be individually targeted and described [26] 
and the third focus on properties based on the frequency domain (like Fourier spectrum [27] or Wavelets).

The statistical approaches do not explicitly seek to understand the hierarchical structure of the fabric texture, but try to represent the same indirectly by non-deterministic properties which define distributions between the gray levels of pixels belonging to an image (first order features) [28]. This approach has the great advantage of low computational cost and main disadvantages the loss of spatial information and the relationship of intensity between neighboring pixels. This method only considers the gray intensity level. The features extracted were the mean and the standard deviation value, asymmetry, kurtosis, energy and entropy [28].

To perform a correct extraction of the information, the choice of the color model is a key step to achieve a good result. Many researchers in the computer vision field recommend using the HSI color model in uncontrolled light conditions due the separation between the color and intensity [29]. Hence, the first step was directed to test the six features mentioned earlier using two different models of colors: RGB (stands for red, green, and blue) and HSI (stands for hue, saturation, and intensity), using the three histograms channels.

Table 1 First Simulation Results

\begin{tabular}{lll}
\hline & RGB & HSI \\
\hline SVM & $89.91 \%$ & $93.42 \%$ \\
ANN & $60.31 \%$ & $55.71 \%$ \\
\hline
\end{tabular}

Analyzing the results for this first simulation, that are presented in Table 1 , it is possible to see that there is no major advantage of using HSI in our case study. Success rates were relatively similar, therefore it was decided to continue to use both color models in the next simulations. It is also possible to see the better performance of the SVM classifier over the ANN.

\subsection{Filters and Co-Occurrence Matrix}

Using the same procedure adopted in [30], the initial RGB/HSI image was converted to grey scale (monochrome). Moreover, and after the application of a Gaussian filter used in a pre-processing stage, was introduced an equalization procedure to obtain the images histogram. This allowed a simplification of the subsequent image processing and respective analysis of results.

In this simulation was also introduced the gray level co-occurrence matrix (GLCM), considered a powerful method by other state of the art authors [15]. The matrix obtained by GLCM considers the relationship between two pixels, i.e., the relationship between a so-called reference pixel and its neighbor. For this reason the features extracted from this matrix are designated secondorder statistics. The co-occurrence in its general form can be specified by 
an absolute frequency matrix, in which two elements of neighboring fabric texture, separated by a specified distance in an orientation, occur in the image. This distance causes a limitation in this approach since there is no automatic method to tune this parameter, requiring an additional computational effort to find a good solution.

The common directions used are: 0, 45, 90 and 135 degrees, and also the matrix obtained from the average of the previous forth matrices directions. Using a sample of this case study an example of a GLCM calculated for the four directions is represented in Figure 6. For an easier illustration the image was reversed, i.e., the white dots correspond to zeros and the black to ones.

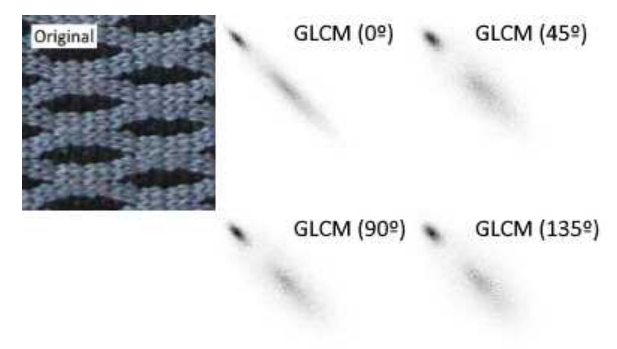

Fig. 6 Representation of GLMC in the fourth directions.

Haralick et al. proposed in [28] fourteen descriptors based on GLCM for fabric texture analyses. According to several authors, six of these measures are most relevant in describing the same: energy, correlation, homogeneity, contrast, entropy and variance. Additionally to these features was also introduced the anisotropy [22], which represents the measure of the symmetry of the intensity values and can be obtained using the following expression:

$$
\text { ani }=\frac{\sum_{i=0}^{k} h(i) * \log _{2}(h(i))}{\text { entropy }}
$$

With $k$ equal to the smallest value so that:

$$
\sum_{i=0}^{k} h(i) \geq 0.5
$$

After the performance of some initial tests the variance feature was removed since it did not had significant impact on the classification ratio.

In uncontrolled light environments is easy to have noise in the captured images. To remove, or at least reduce its influence, different filters were tested. Moreover, to increase the performance of the system some of the filters were utilized to accentuate the differences between the classes.

Filtering operations can be performed both in the space as in the frequency domain. In this work only spatial filters were used. 
Filters are also usually classified into three categories: low-pass, band-pass and high-pass. The low-pass filter attenuates high frequencies, which are related with the detailed information in the image, and also tends to reduce the effect of noise. The high-pass filter, by contrast, enhances detail in the image. A band-pass filter enhances a certain range of frequencies of grey level.

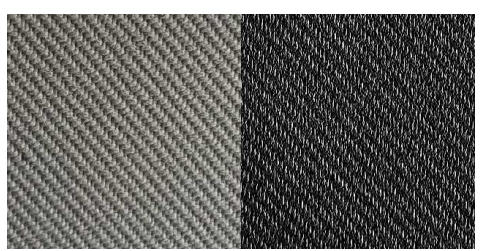

Fig. 7 Example of Laws Filter Implementation.

There are different types of filters:

- Average filter - is a low-pass filter that replaces the value of the central pixel by averaging the pixel values in the mask.

- Gaussian filter is also a low-pass filter. The coefficients of the mask are derived from a two-dimensional Gaussian function.

- Laws Filter measures the energy of texture. It was developed by Laws [31] and has been widely used in fabric textures analysis $[32,33]$. An example of this filter is showed in Figure 7.

- Sobel Filter highlights the lines of the images acting as a gradient operator, computing the magnitude of the gradient as the difference of weighted values of the gray levels of the image. An example is presented in Figure 8 .

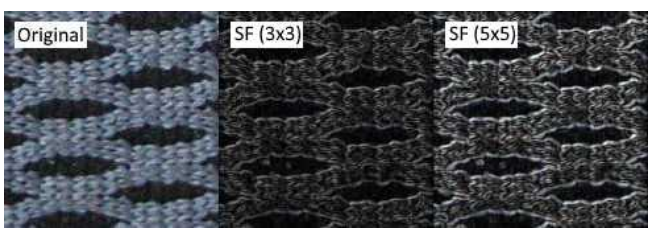

Fig. 8 Example of Sobel Filter Implementation.

As was mentioned earlier the filters have various sizes and types of mask. Those can be applied in different sequential combinations. To find the best combination of filters was simulated the introduction of different types of filters and different sequences of the same. Initially were applied all kinds of filters and was varied the size of the masks, concluding that the optimal mask size was $3 \times 3$. In a second step the size of mask was fixed and the filters that do not contribute successfully to the classification were removed. Based on these simulations was decided to use a mask with the size of $3 \times 3$ pixels and the Law 
Filters of type "le" and "ls". Sobel Filter was also added to highlight the rows and details of fabrics, applying the GLCM in each of four directions in the image obtained.

At the end of this process, and after several simulations was decided to withdraw the characteristics of asymmetry, kurtosis, energy and entropy of the histogram, given the delays in the calculation and low impact on the classification rate. The results presented in Table 2 were obtained using the following set of features: mean and standard deviation of the RGB/HSI histogram (described in section 4.1); energy, correlation, homogeneity, contrast, entropy and anisotropy based on average GLCM with Law Filters of type "le" and "ls"; energy, correlation, homogeneity, contrast, entropy and anisotropy based on GLCM in the four directions mentioned earlier with Sobel Filter.

Table 2 Second Model Simulation Results

\begin{tabular}{lll}
\hline & RGB & HSI \\
\hline SVM & $95.83 \%$ & $93.64 \%$ \\
ANN & $89.91 \%$ & $72.15 \%$ \\
\hline
\end{tabular}

As it is possible to see in Table 2, the results show an improve on the classification ratio ( specially for the ANN classifier) with the introduction of the image pre-processing filtering step and the new set of features. The SVM classifier maintains a better performance, however this time using the RGB color space instead of the HSI.

\subsection{Pyramidal Analysis}

The pyramidal or multi-scale images technique is a simple approach proposed by Burt and Adelson [34] to analyze images at different resolutions.

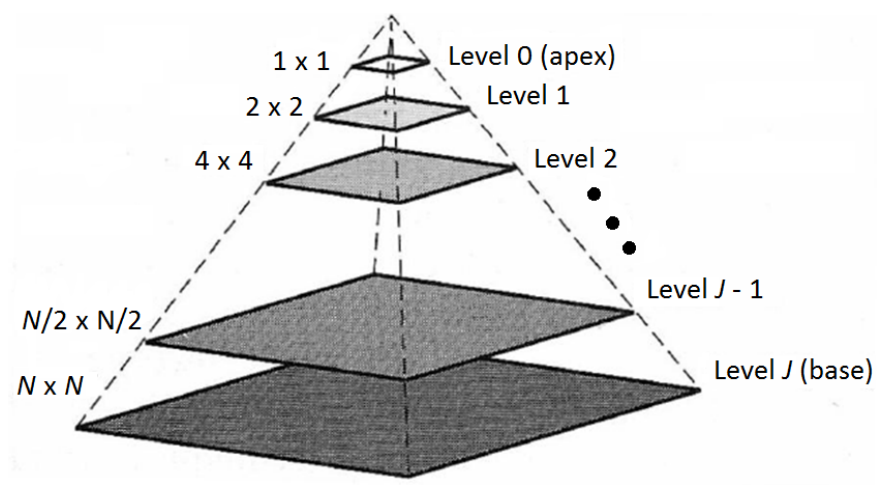

Fig. 9 Pyramidal Analysis [29]. 
It consists in a set of images with decreasing resolution organized in a pyramid shape. The base of the pyramid contains the original image with the maximum resolution, and the following images will successively have lower resolution and size. A scheme of this technique is present in Figure 9. This technique allows to capture unique characteristics of fabric textures at different levels of resolution, that were not accessible in the original image.

Different levels of a pyramidal analysis were tested, concluding that the optimum value was 4 for the presented case study. The classification results obtained for this model are presented in Table 3.

Table 3 Third Model Simulation Results

\begin{tabular}{lll}
\hline & RGB & HSI \\
\hline SVM & $98.46 \%$ & $95.83 \%$ \\
ANN & $94.52 \%$ & $90.79 \%$ \\
\hline
\end{tabular}

Note that, although the improvement verified in the classification results, the growing of the feature vector over the previous model increased the computational time for the classification phase, as is visible in Figure 15 and will later be discussed. The SVM classifier with the RGB image color space is still presenting the best classification ratio.

\section{FEATURES SELECTION}

At this time the classification process use 152 features, and despite the good classification rate from SVM classifier was necessary to find a balance between the classification rate and the time consuming. Therefore, a simple filter selection designated by correlation coefficients was implemented followed by the application of a confusion matrix to certificate the results.

\subsection{Correlation Coefficients}

The correlation coefficients method computes a simple score that measures how informative (correlation) each feature is about the class label. The normalized correlation coefficients for the 152 features are visible in Figure 10.

Based on the achieved results it was tested the reduction of features using only those that have more than 0.3 of weight. This causes a decrease in the number of features, to a total of 22 :

- Pyramid level 1 - energy and homogeneity based on average GLCM with Law Filters of type "ls"; correlation and contrast based on GLCM in one direction (90 degrees); 


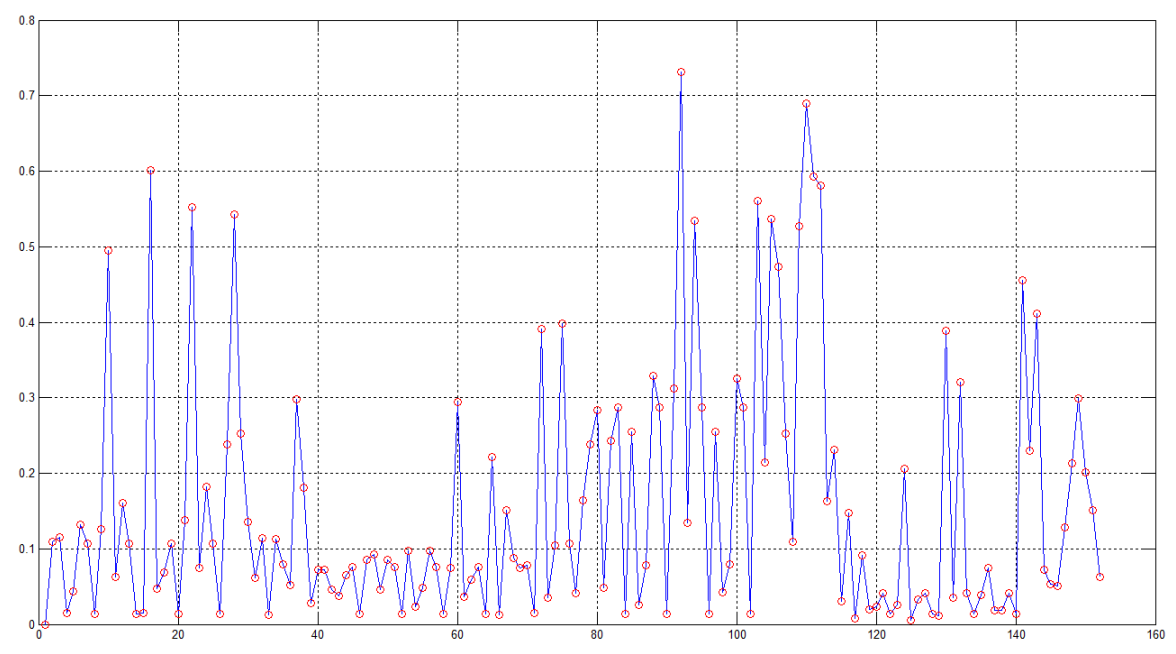

Fig. 10 Features Correlation Results (Weight vs Feature).

- Pyramid level 2 - energy, homogeneity and contrast based on average GLCM with Law Filters of type "ls"; energy, correlation, homogeneity and contrast based on average GLCM with Law Filters of type "le"; correlation based on GLCM in two directions (45 and 90 degrees); contrast based on GLCM in two directions (90 and 135 degrees); energy based on GLCM in one direction (90 degrees);

- Pyramid level 3 - correlation and entropy based on average GLCM with Law Filters of type "le";

- Pyramid level 4 - correlation based on average GLCM with Law Filters of type "ls"; correlation based on GLCM in three directions (45, 90 and 135 degrees);

The results of each classifier are presented in the Table 4.

Table 4 Fourth Model Simulation Results

\begin{tabular}{lll}
\hline & RGB & HSI \\
\hline SVM & $96.27 \%$ & $95.18 \%$ \\
ANN & $89.25 \%$ & $87.28 \%$ \\
\hline
\end{tabular}

Analyzing the results, it is notorious the decrease in the classification ration of both classifiers. Despite this reduction it was achieved some benefits in terms of time consuming on the classification phase, Figure 15. This fact is important, especially in case of a real work cell, where the algorithm's classification rate can be improved with a bigger training sample. 


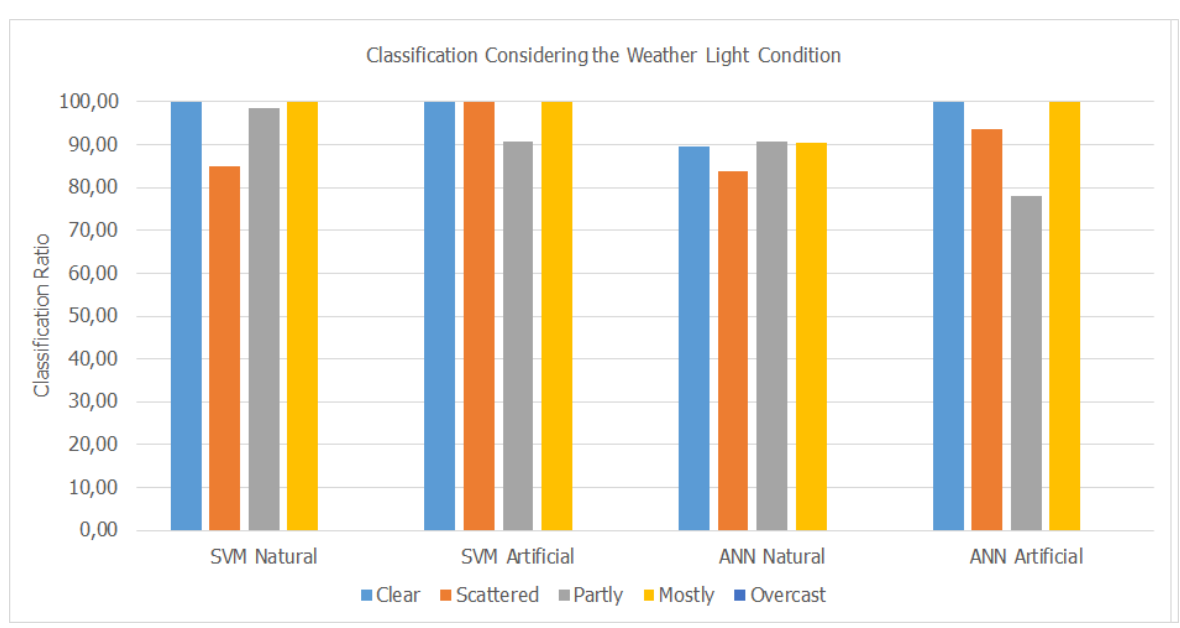

Fig. 11 Classification Ratio of the classifiers according to the weather and light condition.

Figure 11 presents the classification ratio of this simulation model for both classifiers, SVM and ANN, grouped by lighting type and considering the different weather conditions.

Analyzing this Figure, it is possible to see the better performance of the SVM classifier over the ANN. Furthermore, a similar classification ratio is achieved by SVM considering the two different light conditions - natural and artificial. These results show the effectiveness of the proposed pre-processing techniques and extracted features in reducing the effect of the changes on the environment light. Moreover, for samples with natural light the SVM classifier has more difficulty to distinguish the samples taken with scattered weather (85\% of classification ratio), whereas considering the samples with artificial light the SVM classifier has more difficulty to distinguish the samples extracted with partly weather ( $93 \%$ of classification ratio).

\subsection{Confusion Matrix}

The confusion matrix is a very simple and practical technique for analyzing the results of the classification algorithm and assist in the selection or rejection of characteristics of images for the classification process, during construction and optimization algorithm. It consists of a square with equal number of classes in each dimension. At the beginning of the classification process it will consist of zeros. Each classified image will enter this array at position (Correct Class, Class Classified) or vice versa. Thus, an algorithm shall have for optimal classification a diagonal matrix, or as close to it as possible. For a correct analysis of the achieved results two confusion matrix are present.

In the Figures 12 are showed the results of the last simulation (third model) without the selection of features (only for the SVM classifier which proved to be the better choice). 
Considering the results for the SVM classifier and looking for the RGB color space, in 456 classification samples only 7 were mismatched. This represents an error of about $1.5 \%$. While for the HSI color space 19 samples were misclassified (an error of about $4 \%$ ). Moreover, it is possible to see a non negligible confusion classifcation between class 1 and 2 in the HSI color space based model.
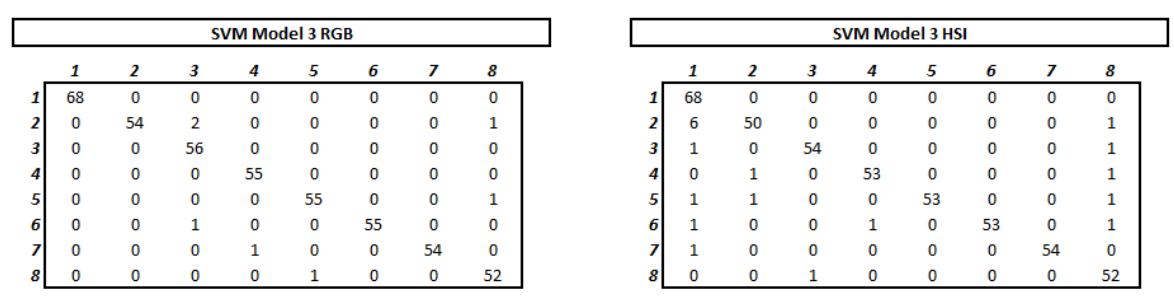

Fig. 12 Third simulation confusion matrix - SVM model.

On the other hand, Figure 13 shows the results of the last simulation with the features selection procedure. For the RGB based model, in 456 classifications 17 were mismatched. About 3.7\% of the results. For the HSI color space, was mismatched 22 samples, about $5 \%$ of classification error. In this last case, it is clear the difficulty of the classifier to distinguish the fabric textures types when compared with class 8 .

This difference could indicate that the images are in bad condition or that some particular details in the training sample make this happen and so the sample training must be bigger to prevent this.

\begin{tabular}{|c|c|c|c|c|c|c|c|c|}
\hline \multicolumn{9}{|c|}{ SVM Model 4 RGB } \\
\hline & 1 & 2 & 3 & 4 & 5 & 6 & 7 & 8 \\
\hline 1 & 68 & 0 & 0 & 0 & 0 & 0 & 0 & 0 \\
\hline 2 & 0 & 57 & 0 & 0 & 0 & 0 & 0 & 0 \\
\hline 3 & 0 & 0 & 54 & 0 & 1 & 0 & 0 & 1 \\
\hline 4 & 0 & 0 & 1 & 52 & 2 & 0 & 0 & 0 \\
\hline 5 & 0 & 2 & 0 & 0 & 54 & 0 & 0 & 0 \\
\hline 6 & 0 & 0 & 4 & 0 & 0 & 52 & 0 & 0 \\
\hline 7 & 0 & 1 & 2 & 0 & 2 & 0 & 50 & 0 \\
\hline 8 & 0 & 1 & 0 & 0 & 0 & 0 & 0 & 5 \\
\hline
\end{tabular}

\begin{tabular}{|c|c|c|c|c|c|c|c|c|}
\hline \multicolumn{9}{|c|}{ SVM Model $4 \mathrm{HSI}$} \\
\hline & 1 & 2 & 3 & 4 & 5 & 6 & 7 & 8 \\
\hline 1 & 65 & 1 & 0 & 0 & 0 & 0 & 0 & 2 \\
\hline ] & 0 & 52 & 0 & 1 & 0 & 0 & 0 & 4 \\
\hline 3 & 0 & 0 & 53 & 0 & 0 & 0 & 0 & 3 \\
\hline 4 & 0 & 2 & 0 & 51 & 0 & 0 & 0 & 2 \\
\hline 5 & 0 & 0 & 0 & 0 & 54 & 0 & 0 & 2 \\
\hline 6 & 0 & 0 & 1 & 0 & 0 & 54 & 0 & 1 \\
\hline 7 & 0 & 0 & 1 & 0 & 0 & 0 & 53 & 1 \\
\hline 8 & 0 & 0 & 0 & 1 & 0 & 0 & 0 & 5 \\
\hline
\end{tabular}

Fig. 13 Fourth Simulation Confusion Matrix - SVM model.

\section{SYSTEM RESULTS}

In this section it is analyzed the key elements of this system, namely the time consuming by the classifier and the success recognition of the same. 


\subsection{Samples Representativeness}

To prove the point mentioned in the previous sections some simulations were performed. In these simulations were used half of the training samples/images and the test sample was not changed. The results presented in Table 5, specially for the case of the ANN classifier, demonstrate the importance of the classifier training step. The SVM classifier proves to be less sensible to the changes performed in this parameter.

At this level of detail the general images will be correctly classified but samples with more detail will fail. To overcome these problems the images samples should be as representative as possible of the variability of each class.

Table 5 Fifth Model Simulation Results

\begin{tabular}{lll}
\hline & RGB & HSI \\
\hline SVM & $96.27 \%$ & $95.18 \%$ \\
ANN & $85.31 \%$ & $82.89 \%$ \\
\hline
\end{tabular}

\subsection{Processing Time Evaluation}

In this section is summarized the time needed by each classifier to perform the different simulations (Figure 14 and Figure 15).

The main restriction of the case study is the speed requirement in the classification stage, because the training stage can be performed off-line, i.e., without stopping the assembly line. The training can be done earlier and saved to disk or memory to be read later for classification stage.


Fig. 14 Simulation Training Times.

In both graphs is possible to see that, for the training step, there is no direct correlation between the number of features and the time needed to train the classifier. Rather, it can be related with the speed of convergence of the classifiers that may be an indication of the quality of the features. 

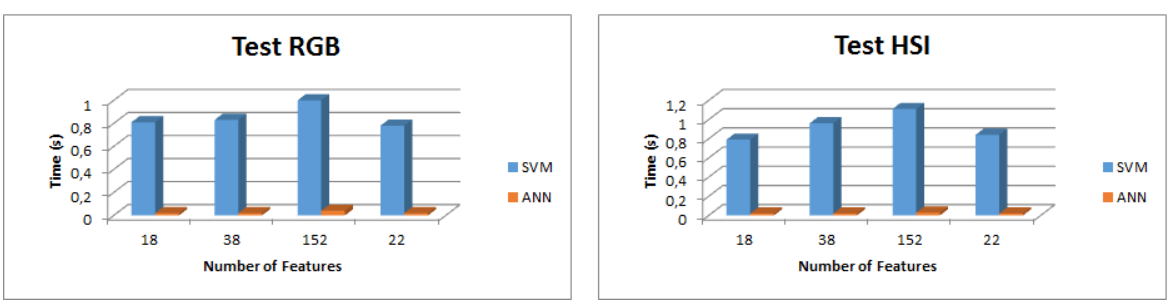

Fig. 15 Simulation Classification Times.

Also it is possible to see that the classification times grows with the number of features, as was expected. Notoriously, this effect is more evident in the case of the SVM classifier.

\section{CONCLUSIONS}

In this case study, a comparison was performed between two different classifiers (ANN and SVM) for an industrial texture recognition problem. The comparison shows the more sensibility of the ANN classifier in respect to the number of features, small number of features translates into low accuracy, but a large number of features can cause over fitting. The SVM proved to be more robust, in this case study, but takes more time in the classification process.

Using only the basic features of the histograms of the three RGB channels, it was possible to achieve a classification rate around $91 \%$ with SVM algorithm. Adding Laws and Sobel filters and calculating their co-occurrence matrices, it was possible to achieve around $94 \%$ per cent of success. In last, and adding the pyramid analyze, the classification rate grows to almost $98 \%$. The filters to highlight textural characteristics, and the use of co-occurrence matrices and extraction of its main features, have proven to be very effective considering the industrial problem in hand.

The application of correlation coefficients for feature selection created a more balanced result in terms of time and success rate. Despite the drop of $2 \%$ on this last simulation, the reduction of the time consumed is much more beneficent to the system. Moreover, the success rate can improve with a more wide sampling training.

This work focus on the usage of a simple methodology with the aim of presenting a mean to solve an industrial case study, but also with a view to their applicability in other similar cases.

The use of approaches such as the LBP and its extensions, FFT, Gabor filters, wavelet transforms and matrices of three-dimensional co-occurrence could be studied and used in situations in which the methods presented in this study do not reach satisfactory results. These methods have been widely studied and used successfully in the area of classification and segmentation of fabrics. 


\section{References}

1. Aksoy, M.S. and Torkul, O. and A Cedimoglu, I.H., An industrial visual inspection system that uses inductive learning, Journal of Intelligent Manufacturing, 2004, v.15, pp. 569-574

2. Rocha, L.F. and Malaca, P. and Silva, J. and Moreira, A.P. and Veiga, G., Development of a 3D model based part recognition system for industrial applications: Main challenges, Industrial Technology (ICIT), 2015 IEEE International Conference on, Pages 3296-3301, March 2015

3. Rocha L.F. and Ferreira M. and Santos V. and Moreira A.P., Object recognition and pose estimation for industrial applications: A cascade system, Robotics and ComputerIntegrated Manufacturing, Volume 30, Issue 6, Pages 605-621, December 2014.

4. Yong-Ren Pu, Li-Wei Chen, Su-Hsing Lee, 2014. Study of Moving Obstacle Detection at Railway Crossing by Machine Vision. Inform. Technol. J., 13: 2611-2618.

5. Brosnan, T. and Sun Da-W. , Improving quality inspection of food products by computer visiona review, Journal of Food Engineering, Volume 61, Issue 1, January 2004, Pages 3-16

6. Veiga G. and Silva C. and Arajo R. and Pires N. and Siciliano B., The ECHORD project proposals analysis Research profiles, collaboration patterns and research topic trends. Expert Syst Appl. 2013 Dec 1;40(17):713240.

7. Theodoridis S. and Koutroumbas K., Academic Press, Boston, 2009.

8. P.J. Fellows, In Woodhead Publishing Series in Food Science, Technology and Nutrition, edited by P.J. Fellows, Woodhead Publishing, 2009, Pages 1-8, Food Processing Technology (Third edition), ISBN 9781845692162, http://dx.doi.org/10.1533/9781845696344.1.

9. Pinto N, Cox DD, DiCarlo JJ (2008) Why is Real-World Visual Object Recognition Hard? PLoS Comput Biol 4(1): e27. doi: 10.1371/journal.pcbi.0040027

10. Alberto Zorcolo and Gustavo Escobar-Palafox and Rosemary Gault and Robin Scott and Keith Ridgway,Study of Lighting Solutions in Machine Vision Applications for Automated Assembly Operations, IOP Conference Series: Materials Science and Engineering,volume 26,pp 12-19,2011.

11. S. Ghorai, A. Mukherjee, M. Gangadaran and P. K. Dutta, "Automatic Defect Detection on Hot-Rolled Flat Steel Products," in IEEE Transactions on Instrumentation and Measurement, vol. 62, no. 3, pp. 612-621, March 2013.

12. Kotsiantis S. B., Supervised Machine Learning: A Review of Classification Techniques. In Proceedings of the 2007 conference on Emerging Artificial Intelligence Applications in Computer Engineering: Real Word AI Systems with Applications in eHealth, HCI, Information Retrieval and Pervasive Technologies, Ilias Maglogiannis, Kostas Karpouzis, Manolis Wallace, and John Soldatos (Eds.). IOS Press, Amsterdam, The Netherlands, The Netherlands, 3-24.

13. Jeon, B. S. and Bae, J. H. and Suh, M. W., Automatic Recognition of Woven Fabric Patterns by an Artificial Neural Network. Textile Research Journal, 73 (7), 645-650., 2013 doi:10.1177/004051750307300714

14. Ben Salem, Y. and Nasri, S., Automatic recognition of woven fabrics based on texture and using SVM, Journal Signal, Image and Video Processing, 429-434 , 2010

15. Loke K. S. (2009). An Approach to Textile Recognition, Pattern Recognition, Peng-Yeng Yin (Ed.), ISBN: 978-953-307-014-8, InTech, DOI: 10.5772/7531. Available from: http://www.intechopen.com/books/pattern-recognition/an-approach-totextile-recognition

16. Kwak, Choonjong and Ventura, Jose A. and Tofang-Sazi, Karim, A neural network approach for defect identification and classification on leather fabric. Journal of Intelligent Manufacturing, 2000,v. 11, pp.485-499.

17. Ahmed S.A. and Dey S. and Sarma K. K. Image texture classification using Artificial Neural Network (ANN). 2011 2nd National Conference on Emerging Trends and Applications in Computer Science (NCETACS). 2011. p. 14.

18. Li S. and Kwok J.T. and Zhu H. and Wang Y. Texture classification using the support vector machines. Pattern Recognition, 2003 Dec;36(12):288393.

19. Sanders D. and Lambert G. and Pevy L. and Tewkesbury G., Improving robotic welding in the shipbuilding industry through the recognition of ship-building parts by pre-locating corners in images. Budapest; 2009 [cited 2014 Jan 7]. Available from: http://eprints.port.ac.uk/5305/ 
20. Pinto A. M. and Rocha L.F. and Moreira A. P. , Object Recognition Using Laser Range Finder and Machine Learning Techniques. Robot Comput-Integr Manuf. 2013 Feb;29(1):1222.

21. Pavelka A. P.,. Procházka P. Algorithms for initialization of neural network weights. Proc. Conf. Tech Comput 2004;

22. MVTec HALCON 10 - Highlights of version 10 [Internet]. [cited 2014 Jan 7]. Available from: http://www.halcon.com/halcon/version10/

23. AForge.NET:: Computer Vision, Artificial Intelligence, Robotics [Internet]. [cited 2014 Jan 7]. Available from: http://www.aforgenet.com/

24. Palm C., Color texture classification by integrative Co-occurrence matrices, Pattern Recognition, Volume 37, Issue 5, May 2004, Pages 965-976, ISSN 0031-3203

25. Haralick, R.M. and Shanmugam, K.and Dinstein, Its'Hak, "Textural Features for Image Classification," in Systems, Man and Cybernetics, IEEE Transactions on , vol.SMC-3, no.6, pp.610-621, Nov. 1973

26. Ballard DH, Brown CM. Computer vision. Prentice-Hall; 1982.

27. Arun D. K.. 1993. Artificial Neural Networks for Image Understanding (1st ed.). John Wiley \& Sons, Inc., New York, NY, USA.

28. Schwartz, W.R., Pedrini, H.: Color Textured Image Segmentation Based on Spatial Dependence Using 3D Co-occurrence Matrices and Markov Random Fields. In: 15th International Conference in Central Europe on Computer Graphics, Visualization and Computer Vision, Plzen, Czech Republic, pp. 8187 (2007)

29. Gonzalez RC, Woods RE. Digital Image Processing (3rd Edition). Upper Saddle River, NJ, USA: Prentice-Hall, Inc.; 2006.

30. Ojala T. and Pietikinen M. and Nisula J. Determining composition of grain mixtures by texture classification based on feature distributions. International Journal of Pattern Recognition and Artificial Intelligence 1996;10:7382.

31. Laws, K. I., Textured Image Segmentation, PhD Thesis, University of Southern California Los Angels Image Processing Institute [cited 2014 Jan 4]. Available from: http://www.dtic.mil/docs/citations/ADA083283

32. Sharma M. and Singh S., Evaluation of texture methods for image analysis. Intelligent Information Systems Conference, The Seventh Australian and New Zealand 2001. 2001. p. 11721.

33. Shenbagavalli R. and Ramar K., Classification of Soil Textures Based on Laws Features Extracted from Preprocessing Images on Sequential and Random Windows, Bonfring International Journal of Advances in Image Processing, pp. 15-18, Decembre 2011.

34. Burt PJ, Adelson EH. The Laplacian Pyramid as a Compact Image Code. IEEE Trans Commun. 1983;31(4):53240.

35. Hosseini, S., Al Khaled, A., Vadlamani, S. Hybrid imperialist competitive algorithm, variable neighborhood search, and simulated annealing for dynamic facility layout problem. Neural Computing and Applications, 2014, 25 (7-8), 1871-1885.

36. Aleksander, Igor, and Helen Morton. An introduction to neural computing. Vol. 3. London: Chapman \& Hall, 1990.

37. Burges, Christopher J.C. , A Tutorial on Support Vector Machines for Pattern Recognition, Data Mining and Knowledge Discovery, v.2, Pages 121-167, 10.1023/A:1009715923555,1998 